Proceeding Paper

\title{
Building Extraction from Very High Resolution Stereo Satellite Images Using OBIA and Topographic Information ${ }^{\dagger}$
}

\author{
Minakshi Kumar* and Ashutosh Bhardwaj \\ Photogrammetry and Remote Sensing Department, Indian Institute of Remote Sensing, Dehradun, \\ Uttarakhand 248001, India; ashutosh@iirs.gov.in \\ * Correspondence: minakshi@iirs.gov.in; Tel.: +91-135-252-4118 \\ † Presented at the 3rd International Electronic Conference on Geosciences, 7-13 December 2020; \\ Available online: https://iecg2020.sciforum.net/.
}

Citation: Kumar, M.; Bhardwaj, A. Building Extraction from Very High Resolution Stereo Satellite Images Using OBIA and Topographic Information. Environ. Sci. Proc. 2021, 5, 1. https://doi.org/10.3390/ IECG2020-08908

Academic Editor: Jesus Martinez Frias

Published: 7 December 2020

Publisher's Note: MDPI stays neutral with regard to jurisdictional claims in published maps and institutional affiliations.

Copyright: $@ 2020$ by the authors. Licensee MDPI, Basel, Switzerland. This article is an open access article distributed under the terms and conditions of the Creative Commons Attribution (CC BY) license (http://creativecommons.org/licenses/by/4.0/).

\begin{abstract}
The availability of very high resolution (VHR) satellite imagery $(<1 \mathrm{~m})$ has opened new vistas in large-scale mapping and information management in urban environments. Buildings are the most essential dynamic incremental factor in the urban environment, and hence their extraction is the most challenging activity. Extracting the urban features, particularly buildings using traditional pixel-based classification approaches as a function of spectral tonal value, produces relatively less accurate results for these VHR Imageries. The present study demonstrates building extraction using Pleiades panchromatic (PAN) and multispectral stereo satellite datasets of highly planned and dense urban areas in parts of Chandigarh, India. The stereo datasets were processed in a photogrammetric environment to obtain the digital elevation model (DEM) and corresponding orthoimages. DEM's were generated at $0.5 \mathrm{~m}$ and $2.0 \mathrm{~m}$ from stereo PAN and multispectral datasets, respectively. The orthoimages thus generated were segmented using object-based image analysis (OBIA) tools. The object primitives such as scale parameter, shape, textural parameters, and DEM derivatives were used for segmentation and subsequently to determine threshold values for building fuzzy rules for building extraction and classification. The rule-based classification was carried out with defined decision rules based on object primitives and fuzzy rules. Two different methods were utilized for the performance evaluation of the proposed automatic building approach. Overall accuracy, correctness, and completeness were evaluated for extracted buildings. It was observed that overall accuracy was higher $(>93 \%)$ in areas having larger buildings and that were sparsely built-up as compared to areas having smaller buildings and being densely built-up.
\end{abstract}

Keywords: object-based image analysis; high resolution image processing; building extraction; textures

\section{Introduction}

Rapid growth in urbanization demands the monitoring of features and a decisionmaking system for planning and utility mapping. Feature extraction is an important aspect of remote sensing and is becoming more important than ever due to the large number of datasets coming each day, which require extensive processing and automation. Subsequently, manual extraction and interpretation are time-consuming. Automatic feature extraction has become the main objective to save time in updating data, and as a result of this, people are changing progressively to new procedures [1], especially focusing on extracting roads [2] and buildings [3,4]. Extraction of buildings on a common platform using high resolution (HR) satellite imagery is a complex and challenging task due to high variability in building features. A wide range of techniques and algorithms have been proposed for automatically constructing 2D or 3D building models from aerial imagery $[5,6]$. 
Quantitative information about building density can serve as a useful tool for the study of land-use changes, illegal building development, urban expansion, etc., and, therefore, can contribute to the sound development of an area. Typical urban structures often have sizes smaller than the spatial resolution of the sensor when they are represented on a medium resolution image. In a high spatial resolution, they are characterized by different texture values them which can be used for identifying buildings and their densities. Rational polynomial coefficients (RPCs) have been a successful alternative to physical sensor models in relating the image space with the ground space $[7,8]$. DEMs are an important requirement for the generation of high-quality orthoimages and the preparation of maps [9-12]. HR images whose spatial resolutions are about $1 \mathrm{~m}$ were assessed for the performance of the generated rational function model (RFM) generation process as well as the physical sensor model. The stereo pair's horizontal positioning accuracy of the physical and RPC models was found to be $5.0 \mathrm{~m}$ in both cases, with a circular error of 90 percent (CE90) [13].

Detecting building objects is a challenging topic even with high-quality remote sensing data. Rooftops have many forms and appearances regarding their color, shape, material, and optical issues such as illumination, reflection, and perspective also bias the automatic identification. Although many studies have focused on feature extraction, they use spatial or structural features or both [14]. There are very few studies on the incorporation of DEM in building extraction. An attempt is made here to utilize DEM and its derivatives, plus textures, along with spectral and spatial information in an object-based environment for building extraction.

\section{Materials and Methods}

VHR and HR satellite Pleiades-1A panchromatic (PAN) and multispectral stereo image datasets were used in this study. Chandigarh is a city and a union territory of India that serves as the capital of the Indian states of Punjab and Haryana. Chandigarh is bordered by the state of Punjab to the north, west, and south, and by the state of Haryana to the east. Chandigarh was one of the early planned cities in post-independence India and is internationally known for its architecture and urban design. The master plan of the city was prepared by Swiss-French architect Le Corbusier. It is located near the foothills of the Shivalik range of the Himalayas in northwest India. The overall methodology for photogrammetric processing and feature extraction carried out in this study is depicted in Figure 1.

Elevations play a significant role in high spatial resolution image photogrammetric processing and orthoimage generation. The acquired Pleiades stereo data was photogrammetrically processed for satellite triangulation using the RPCs. Thereafter, the DEM's were generated for the experimental site using PAN and multispectral stereo pairs at 0.5 $\mathrm{m}$ and $2 \mathrm{~m}$ posting intervals, respectively. The root mean square error (RMSE) achieved for Panchromatic (PAN) and Multi-spectral (MS) stereo datasets was 0.05 pixels and 0.03 pixels, respectively. Further the digital elevation model (DEM) and corresponding orthoimages were generated for both PAN and multispectral stereo datasets.

The two orthoimages were then fused together using principal components. An integrated dataset comprising of resulting Pan sharpened orthoimages, DEM layers, suitable texture layers from DEM, and orthoimages were then segmented using a multiresolution segmentation technique. The object primitives such as scale parameter, shape, textural parameters, DEM derivatives, and textures were used for segmentation and subsequently to determine threshold values for building fuzzy rules for building extraction and classification. The rule-based classification was carried out with defined decision rules based on object primitives and fuzzy rules. 


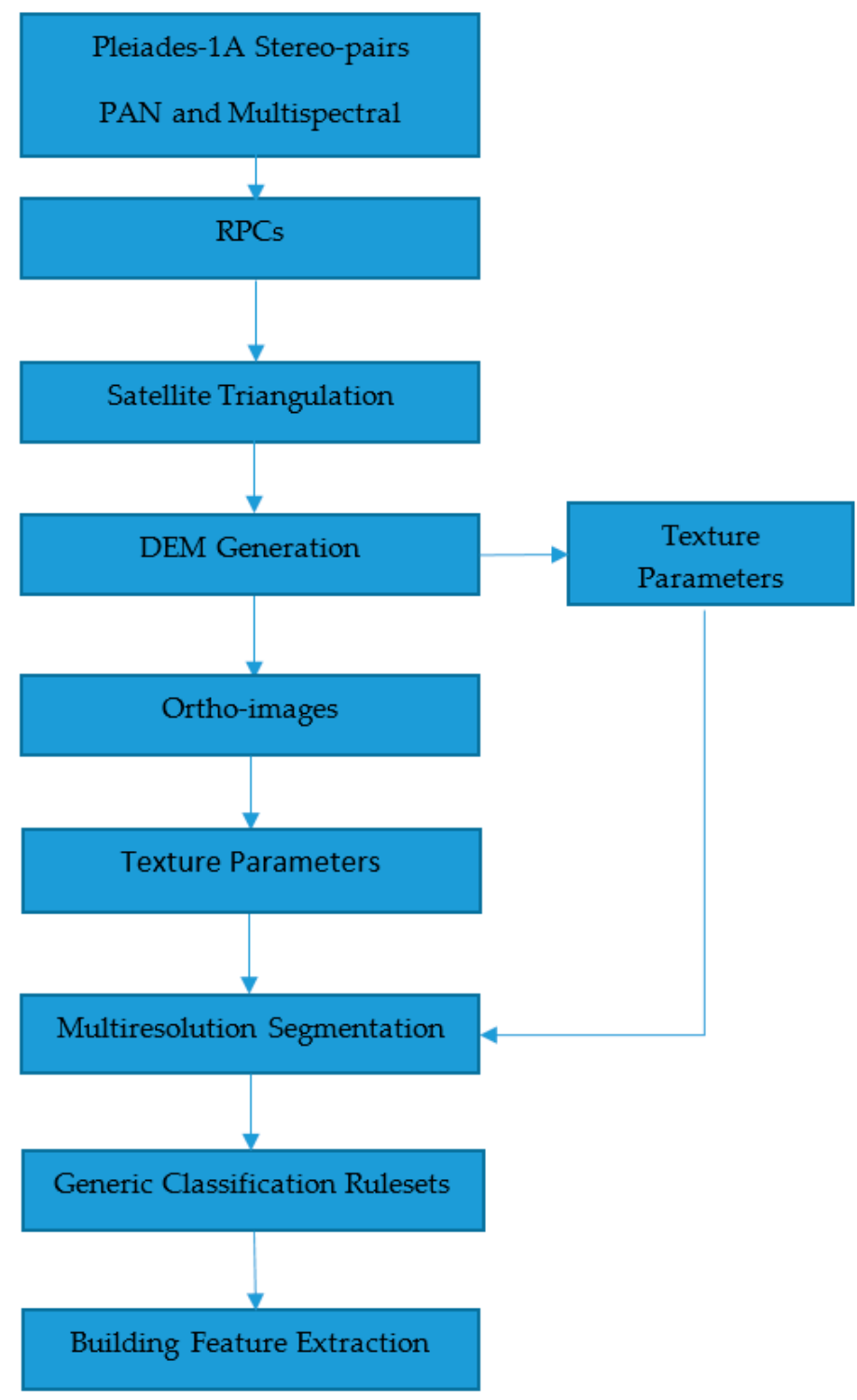

Figure 1. Methodology for photogrammetric processing and feature extraction.

\section{Results and Discussion}

The results are illustrated in Figures 2 and 3. The DEM (Figure 2a) has brighter tones both for buildings and roads. Low elevation areas are in black and agriculture areas and grasslands are grey. It was observed that variance and angular second moment (Figure 2c) were able to separate roads from buildings; variance, entropy and grey level co-occurence matrix (GLCM) correlation (Figure 2d) were useful in extracting building footprints. The PAN sharpened image is presented in Figure 3a, and the variance of the same in Figure $3 \mathrm{~d}$ indicates that buildings and roads are distinguishable from other features using this texture. The multiresolution segmentation using a fractal net evolution approach (FNEA; scale parameter converged to 220), was found suitable for both larger and smaller buildings. Since the main aim was to extract urban features (mainly buildings), vegetation (trees and grassland) was eliminated using a normalized difference vegetation index (NDVI). The quality measures were computed, which addressed the problem of completeness and correctness of the extracted road network. The results obtained were completeness $93.47 \%$ and a correctness of $92.94 \%$, with the overall quality being $90.79 \%$. 


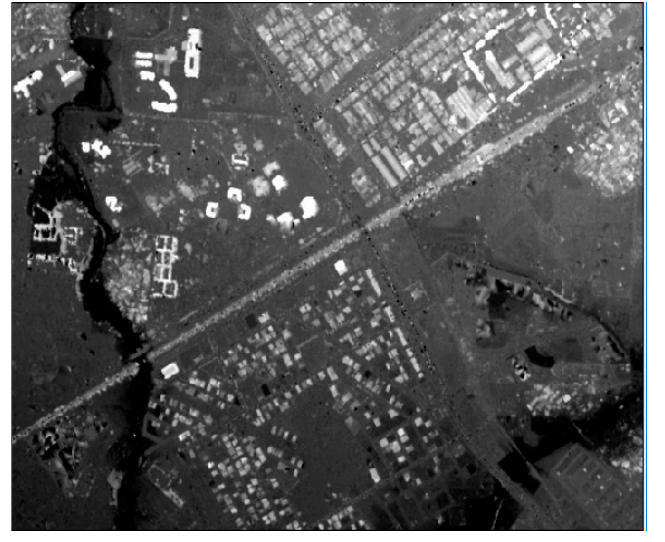

(a)

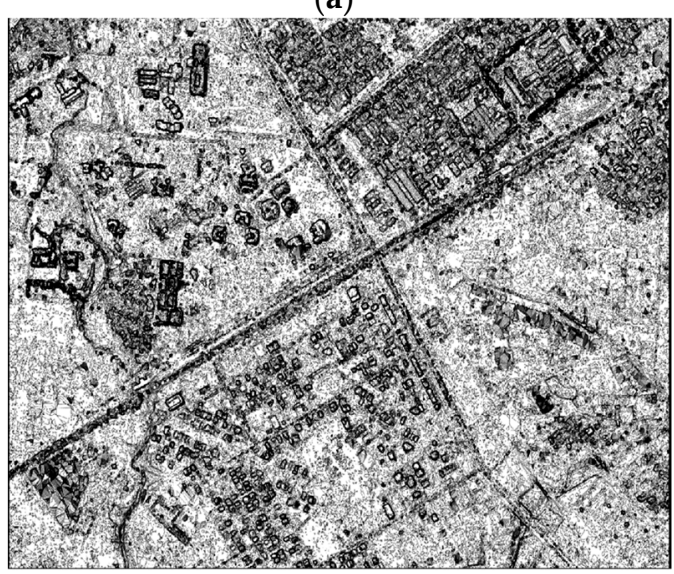

(c)

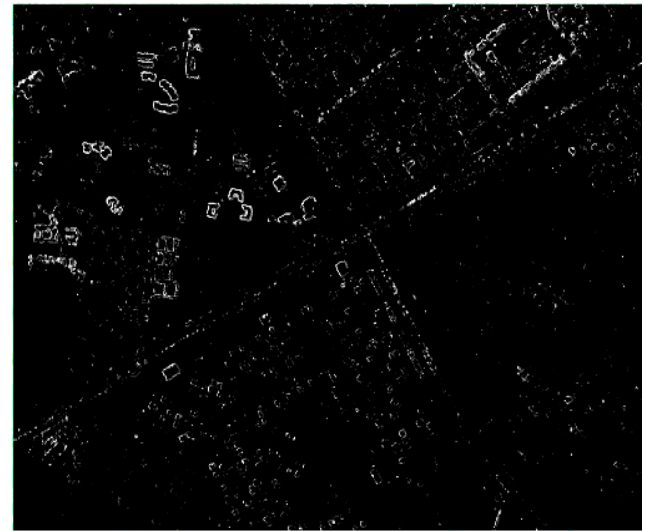

(b)

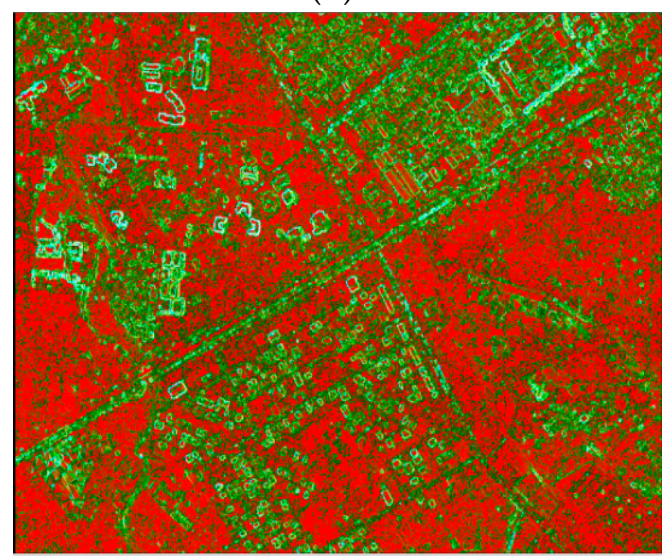

(d)

Figure 2. (a) DEM, (b) variance, (c) angular second moment, and (d) FCC (R: GLCM correlation, G: entropy B: variance).

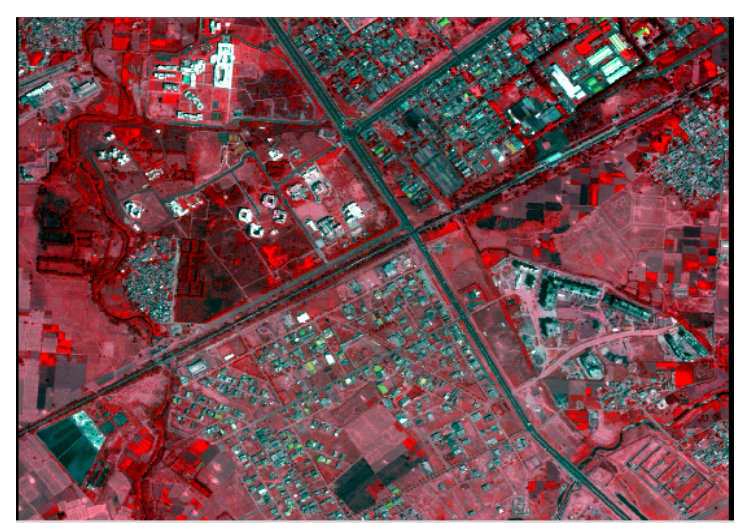

(a)

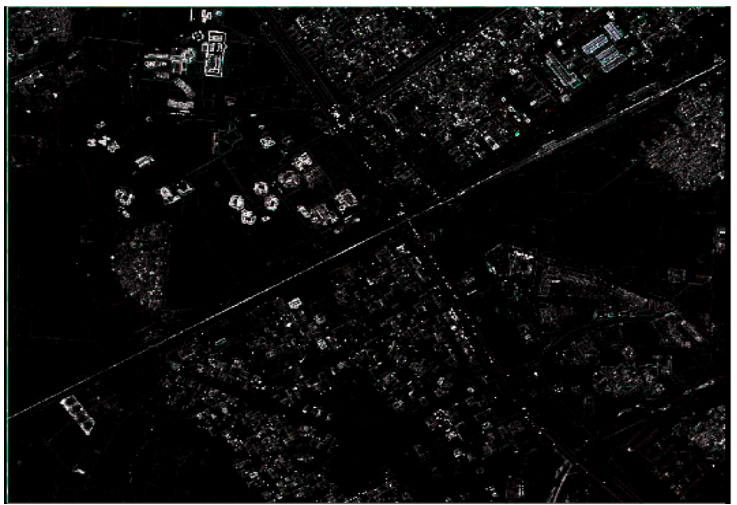

(b) 


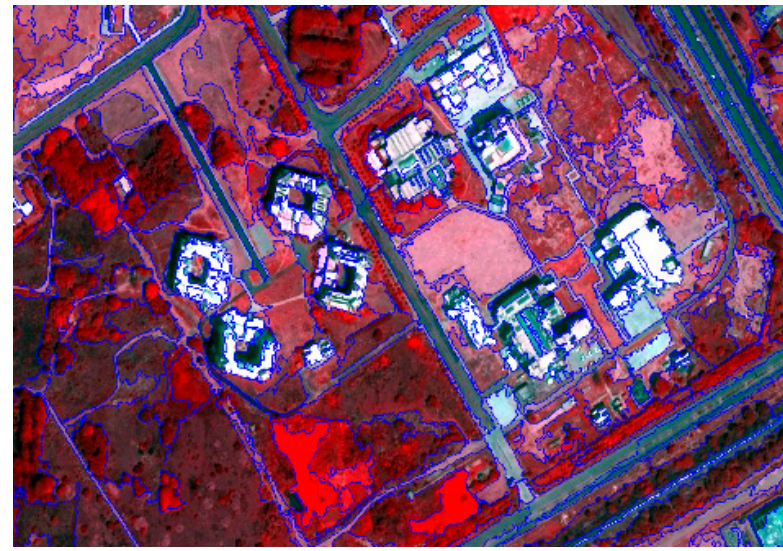

(c)

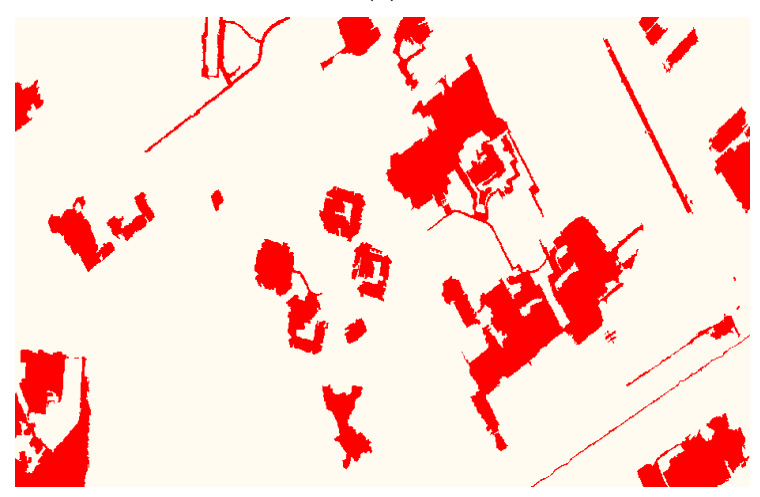

(e)

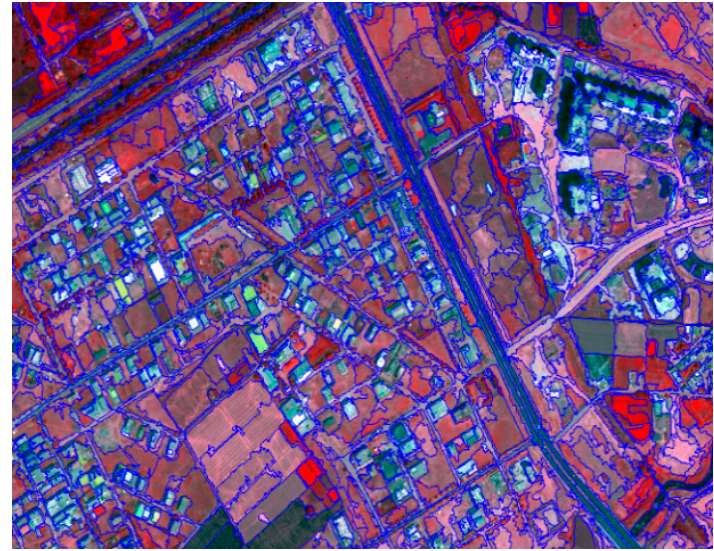

(d)

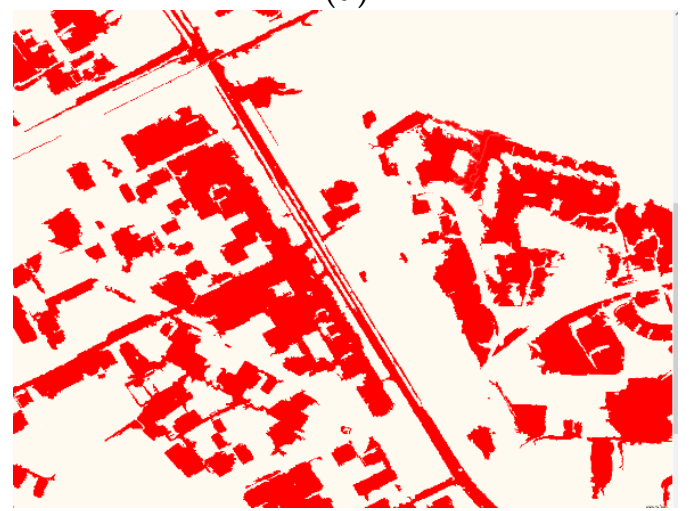

(f)

Figure 3. (a) Fused FCC orthoimage, (b) FCC-GLCM variance, (c,d) segmented images (e,f) extracted buildings.

\section{Conclusions}

As evident from the achieved RMSE values for the satellite triangulation, the bundle block adjustment based on RFM is of good quality. The block is thus able to produce good DEM and orthoimages for the feature extraction as observed from visual interpretation of clearly identifiable drainage networks and built-up regions in the DEM and orthoimages. Generic rulesets, which can be transferable for each and every dataset, can have limitations in terrain areas. Therefore, the rulesets are having restrictions for different feature extraction. Slope, altitude, and the nature of terrain play an important role in urban feature Interpretation. In this paper, an approach to extract buildings and roads from very high resolution orthoimages was carried out using a DEM and various texture parameters extracted from DEM and raw images. The method described above worked well in those areas which also contained shadows. The obstacles encountered were shadows of high buildings falling on smaller building rooftops and the tone of the road spectrally mixing with building rooftops. A higher dimension dataset (texture and transforms) was found useful in most of the problems encountered. It was observed that overall accuracy was higher $(>93 \%)$ in areas having larger buildings and being sparsely built-up as compared to areas having smaller buildings and being densely built-up. The method worked well to extract fine building boundaries both in highly dense and rural areas.

Author Contributions: Both authors conceived, designed, performed the experiments, analyzed the data, and wrote the paper. All authors have read and agreed to the published version of the manuscript.

Funding: This research received no external funding.

Acknowledgments: The authors are highly indebted to Director of IIRS for his continuous support and encouragement in conducting the research activities. 
Conflicts of Interest: The authors declare no conflict of interest.

\section{References}

1. Kumar, M.; Tiwari, P.S.; Pande, H.; Dadhwal, V.K. A semi-automatic segmentation approach procedure for agriculture field boundary extraction in remotely sensed imagery. In Proceedings of the Indian Society of Remote Sensing symposia, Nagpur, India, 16-18 September 2009.

2. Kumar, M.; Singh, R.K.; Raju, P.L.N.;Krishnamurthy, Y.V.N. Road Network Extraction from High Resolution Multispectral Satellite Imagery Based on Object Oriented Techniques. In Proceedings of the ISPRS Annals of the Photogrammetry, Remote Sensing and Spatial Information Sciences, Volume II-8, 2014 ISPRS Technical Commission VIII Symposium, Hyderabad, India, 9-12 December 2014

3. Khatriker, S.; Kumar, M. Building Footprint Extraction From High Resolution Satellite Imagery Using Segmentation. In Proceedings of the ISPRS Annals of the Photogrammetry, Remote Sensing and Spatial Information Sciences, Dehradun, India 20-23, November 2018, doi:10.5194/isprs-archives-XLII-5-123-2018.

4. Kumar, M.; Garg, P.K.; Srivastav, S.K. A Spectral Structural Approach for Building Extraction from Satellite Imageries. Int. J. Adv. Remote Sens. GIS 2018, 7, doi:10.23953/cloud.ijarsg.338.

5. Mayer, H. Automatic object extraction fromaerial imagery a survey focusing on buildings. Comput. Vision Image Underst. 1999, 74, 138-149.

6. Shufelt, J. A.Performance evaluation and analysis of monocular building extraction from aerial imagery. IEEE Trans. Pattern Anal. Machine Intell. 1999, 21, 311-326.

7. Dial, G. IKONOS satellite mapping accuracy. In Proceedings of ASPRS Annual Convention 2000; ASPRS: Washington, DC, USA, 2000, (CD-ROM).

8. Grodecki, J.; Dial, G. Block adjustment of high-resolution satellite images described by Rational Polynomials. Photogramm. Eng. Remote Sens. 2003, 69, 59-68, doi:10.14358/PERS.69.1.59.

9. Alkan, M.; Jacobsen, K. Information content capabilities of very high resolution optical space imagery for updating GIS database. In Image Sensing Technologies: Materials, Devices, Systems, and Applications II; Dhar, N.K., Dutta, A.K., Eds.; 2015; SPIE Digital Library, Volume 9481, p. 94810K, doi:10.1117/12.2177857.

10. Bhardwaj, A.; Enkhtur, B.; Raghavendra, S.; Agrawal, S. Topographic database generation and 3D feature extraction techniques using Cartosat-1 stereo data. Indian Cartogr. J. Indian Natl. Cartogr. Assoc. 2010, 30, 1-6.

11. Bhardwaj, A.; Jain, K.; Chatterjee, R. S. Generation of high-quality digital elevation models by assimilation of remote sensingbased DEMs. J. Appl. Remote Sens. 2019, 13, 1, doi:10.1117/1.JRS.13.4.044502.

12. Nandakumar, R.; Chamy, M. P. T.; Soma, S.; Kopparthi, S.; Paswan, G.; Prakash, S. Synthesis of investigations under ISPRSISRO Cartosat-1 scientific assessment programme primarily for DSM generation. In the International Archives of the Photogrammetry, Remote Sensing and Spatial Information Sciences; Beijing, China, 2008; Volume XXXVII, pp. $1279-1286$.

13. Ye, J.; Lin, X.; Xu, T. Mathematical Modeling and Accuracy Testing of WorldView-2 Level-1B Stereo Pairs without Ground Control Points. Remote Sens. 2017, 9, 737, doi:10.3390/rs9070737, 2017.

14. Kumar, M.; Roy, P.S. Utilizing the Potential of World View -2 for Discriminating Urban and Vegetation Features Using Object Based Classification Techniques. J. Indian Soc. Remote Sens. 2013, 41, doi:10.1007/s12524-012-0257-9. 